American Journal of Applied Sciences 9 (4): 484-491, 2012

ISSN 1546-9239

(C) 2012 Science Publications

\title{
Mulberry Fruit Mitigates Alcohol Neurotoxicity and Memory Impairment Induced by Chronic Alcohol Intake
}

\author{
${ }^{1,2}$ Jintanaporn Wattanathorn, ${ }^{2}$ Nattaporn Phunchago, \\ ${ }^{1,2}$ Supaporn Muchimapura, ${ }^{2}$ Wipawee Thukhum-Mee, \\ ${ }^{3}$ Kowit Chaisiwamongkol, ${ }^{4}$ Wiroje Kaewrueng and ${ }^{4}$ Sathaporn Wongareonwanakij \\ ${ }^{1,2,3}$ Department of Physiology, \\ ${ }^{2}$ Integrative Complimentary Alternative Medicine, \\ Research and Development Group, \\ ${ }^{3}$ Department of Anatomy, \\ Faculty of Medicine, Khon Kaen University, Khon Kaen 40002, Thailand \\ ${ }^{4}$ Department of Sericulture, \\ Ministry of Agriculture and Cooperatives, Bangkok 10200, Thailand
}

\begin{abstract}
Problem statement: To date, the therapeutic strategy efficacy against memory impairment induced by alcohol intoxication is still limited. The novel therapeutic strategy which is easy to approach, less toxic and less cost is required. Based on the role of oxidative stress in memory impairment induced by alcohol, the neuroprotective effect of substance possessing antioxidant has gained much attention. Therefore, we aimed to determine the effect of Morus alba fruits, substance possessing antioxidant, on spatial memory and brain damage in hippocampus. Approach: Male Wistar rats were induced alcoholism by increasing the alcohol concentration in drinking water gradually increased to $30 \%$ within 15 -week period. Then, the alcoholic rats were orally given mulberry fruits powder at doses of 2, 10 and $50 \mathrm{mg} \mathrm{kg}^{-1} \mathrm{BW}$ at a period of 14 days. The memory was assessed using Morris water maze after single administration and every 7 days until the end of the experimental period and at the end of experiment, hippocampus was isolated and determined the neuron density. In addition, the evaluation of Acetylcholinesterase (AChE) activity and Malondialdehyde (MDA) level were also performed. Results: Our results showed that all doses of mulberry fruits enhanced spatial memory and neurons density in hippocampus. The suppression of both AChE activity and MDA level were also observed. These results suggested that the neuroprotection of mulberry fruits might occur partly via the decreased oxidative stress damage while the cognitive enhancing effect might occur partly via the increased hippocampal neuron density and the suppression of AChE activity. Conclusion: Mulberry fruits can protect against brain damage and memory impairment induced by alcoholism. Therefore, mulberry fruits may be served as natural resource for developing food supplement against alcoholism. However, further researches about possible active ingredient and pharmacokinetic are required before moving forward to clinical trial study.
\end{abstract}

Key words: Mulberry fruits, Acetylcholinesterase (AChE), Malondialdehyde (MDA), Cyanidin-3Glucoside Equivalents (CGE), induce impairments, excessive alcohol consumption

\section{INTRODUCTION}

Alcohol is recognized as one of the most commonly abused drugs worldwide. Alcohol has been used as psychoactive drug, but chronic and excessive alcohol consumption can produce various alcoholrelated problem including social, economic and public health problem with profound impacts on brain functions and behaviors.
Chronic ethanol exposures have been shown to induce impairments in a variety of tasks requiring spatial cognition (Arendt et al., 1989; Matthews and Morrow, 2000). The memory impairment induced by chronic alcohol consumption was reported to be associated with the decreased cholinergic system (Arendt et al., 1989; Nordberg et al., 1982). Moreover, it was that chronic alcoholism produced the neurodegeneration and reduction of hippocampal

Corresponding Author: Jintanaporn Wattanathorn, Department of Physiology, Faculty of Medicine, Khon Kaen University, Khon Kaen, 40002, Thailand Tel: +66-43-348394 Fax: +66-43-348394 
volume (Agartz et al., 1999). The mechanism of alcohol-induced brain damage is complex. However, oxidative stress has been reported to be one factor contributing the important role on the neurodegeneration induced by alcohol (Esterbauer et al., 1991). It was reported that ethanol consumption increased oxidative stress both via the increase free radical formation and the decreased antioxidant enzyme activities (Das and Vasudevan, 2007). Based on the crucial role of oxidative stress mentioned earlier, the neuroprotective effect of substances possessing antioxidant has gained much attention.

Mulberry (Morus alba L.) or Mohn in Thai, a plant in family of Moraceae, is widely cultivated in the North and North East of Thailand. Mulberry fruit is used not only as fruit but also as medicine. According to the traditional folklore, mulberry fruit is used to protect against liver and kidney damage, strengthen the joints, improve eyesight and have anti-aging effects ( $\mathrm{Li}$ and Luo, 2003). It is also used for the treatment of sore throat, fever, hypertension and anemia (Ma, 2002; Gong and Zhu, 2008). Recent findings have shown that mulberry fruits can protect against brain damage in various conditions including Parkinson's disease (Kim et al., 2010) and cerebral ischemia (Kang et al., 2006). Moreover, mulberry fruit extract has been reported to decrease $\beta$-amyloid protein leading to the reduction of learning and memory impairment in SenescenceAccelerated Mice (SAMP) (Shih et al., 2010). Although, several researches have already demonstrated the neuroprotective of mulberry fruits, less scientific evidence about the effect of mulberry fruits on hippocampus, the area contributing important role on learning and memory, in memory impairment induced by chronic alcohol consumption is available. Therefore, this present study aimed to determine the effect of mulberry fruits on spatial memory and on brain damage in hippocampus induced by chronic alcohol consumption. In addition, the alteration of $\mathrm{AChE}$ and oxidative damage marker were also further investigated.

\section{MATERIALS AND METHODS}

Preparation of mulberry fruits freeze-dried powder: All mulberry fruits (Morus alba var Chiangmai) used in this study is prepared and provided by The Institute of Queen Sirikit Seri Culture, Thailand. Mulberry fruits were collected from the Queen sirikit seri culture center udon thani. All berries were picked at the commercially ripen stage and selected according to uniformity color. Then, the fruits were dried at $70 \%$ celcius for 4 days and grounded to powder. In addition, the mulberry fruit powders were also kept in dark air tight bottle at $-20^{\circ} \mathrm{C}$. The mulberry fruit powder used in this study contained phenolic compounds at concentration of $519 \pm 0.004 \mathrm{mg}$ $\mathrm{GAE} / \mathrm{g}$ fresh matter of fruit. It also contained anthocyanin at concentration of $634.56 \pm 23.62 \mathrm{mg}$ of Cyanidin-3Glucoside Equivalents (CGE) per $100 \mathrm{~g}$ of fresh weight.

Animal: Adult male Wistar rats, 8 weeks old, were used as experiment animals. They were obtained from National Animal Center, Salaya, Nakornpatom. The weights of the animals on the first day of experiment were 180-220 g. All animals were given access to food and water ad libitum. They were randomly housed $5 \%$ and maintained in 12:12 light: dark cycle and given access to food and water ad libitum. The experiments were performed to minimize animals suffering and the experiment protocols were approved by the Institutional Animal Care and Unit Committee Khon Kaen University, Thailand.

Experimental protocol: All rats were induced alcoholism by means of a semi-voluntary intermittent daily intake of different concentrations of alcohol. In brief, rats were exposed to alcohol in drinking water. The alcohol concentration in drinking water was gradually increased from 5-20\% from the first to fourth week (5\% per week). Then the alcohol concentration was raised to $30 \%$ from the fifth to thirtieth. The animals were assessed tremor by stationary dowel test and aggression as indicators for alcoholism (Gotz et al., 2001). Then the alcoholic rat were divided into 6 groups as following; (1) Vehicle plus ethanol (2) Vitamin C $250 \mathrm{mg} \mathrm{Kg}{ }^{-1} \mathrm{BW}$ plus ethanol (3)-5) Mulberry fruits at doses of 2,10 and $50 \mathrm{mg} \mathrm{Kg}^{-1} \mathrm{BW}$ respectively plus ethanol.

Since the effect of alcohol on oxidative stress and cognitive impairment were focused, the positive control treated groups used in this study were treated with vitamin C (well established antioxidant) and Aricept (a standard drug treated dementia) at doses of 250 and 1 $\mathrm{mg} \mathrm{kg}^{-1} \mathrm{BW}$ respectively. In addition, the animals in group 4-6 were treated with various doses of mulberry fruits ranging from 2,10 and $50 \mathrm{mg} \mathrm{kg}^{-1} \mathrm{BW}$. The animals were assessed the effect of mulberry fruits on cognitive function using Morris water maze test after single intervention, 7 and 14 days of intervention. In order to determine the effect of mulberry fruits on brain damage, all rats were sacrificed and removed the brain for the determination of brain damage after 2 weeks of treatment.

Determination of spatial memory: The spatial memory was determined using Morris water maze test. The water maze was a circular pool with $160 \mathrm{~cm}$ in 
diameter, $60 \mathrm{~cm}$ in height filled with water (approximately $42 \mathrm{~cm}$ deep, temperature of $23-24^{\circ} \mathrm{C}$ ), filled up with tap water for $40 \mathrm{~cm}$ deep and the water surface was covered with nontoxic milk powder. The pool was divided into 4 quadrants and the removable escape platform was placed in the center on one quadrant below the water level. For animals, the location of the platform was invisible and it remained there throughout the training. The animals must memorize the environmental cues to locate the platform. Each animal was placed in the water in the starting quadrant and allowed to swim until it found and climbed onto the platform. The time for animal to reach the hidden platform was recorded as escape latency. Then, $24 \mathrm{hr}$ later, the animals were exposed to the same test except that the hidden platform was removed. The time which the animals spent in the quadrant previously located the platform was recorded as the retention time.

Histological procedure: Following anesthesia with sodium pentobarbital $\left(60 \mathrm{mg} \mathrm{kg}^{-1} \mathrm{BW}\right)$, fixation of the brain was carried out by transcardial perfusion with fixative solution containing $4 \%$ paraformaldehyde in $0.1 \mathrm{M}$ phosphate buffer $\mathrm{pH}$ 7.3. The brains were removed after perfusion and stored over a night in a fixative solution that used for perfusion. Then, they were infiltrated with $30 \%$ sucrose solution for approximately $4^{\circ} \mathrm{C}$. The specimens were frozen rapidly and $30 \mu \mathrm{M}$ thick sections were cut on cryostat. They were rinsed in the phosphate buffer and picked up on slides coated with $0.01 \%$ of aqueous solution of a high molecular weight poly L-lysine.

Morphological analysis: Five coronal sections of each rat in each group were studied quantitatively. Neuronal counts in hippocampus were performed by eye using a $40 \times$ magnification with final field $255 \mu^{-2}$ according to the following stereotaxic coordinates: AP $-4.8 \mathrm{~mm}$, lateral $\pm 2.4-6 \mathrm{~mm}$, depth 3-8 $\mathrm{mm}$. The observer was blind to the treatment at the time of analysis. Viable stained neurons were identified on the basis of a stained soma with at least two visible processes. Counts were made in five adjacent fields and the mean number extrapolated to give total number of neurons per $255 \mu^{-2}$. All data are represented as number of neurons per $255 \mu \mathrm{m}^{2}$

Determination of malondialdehyde level and acetylcholinesterase activity: Hippocampus was isolated and prepared as hippocampal homogenate and the determination of the Malondialdehyde (MDA) level and acetylcholinesterase activity in hippocampus were performed. Malondialdehyde was indirectly estimated by determining the accumulation of Thiobarbituric Acid Reactive Substances (TBARS) while the activity of AChE was determined using.

Statistical analysis: Data are presented as mean \pm Standard Error of Mean (SEM). One-way Analysis Of Variance (ANOVA), followed by Tukey post hoc test. A probability level less than 0.05 were accepted as significance.

\section{RESULTS}

Effect of mulberry fruits on cognitive deficit induced by chronic ethanol intake: We have found that Vitamin C which used as positive control in this study significantly attenuated the increase in escape latency induced by alcohol administration after single, 7 and 14 days after mulberry administration $(\mathrm{p}<0.001,01$ and 0.001 respectively, compared with vehicle treated group) as shown in Fig. 1. However, Vitamin C treatment failed to attenuate the decrease in retention time induced by alcohol consumption as shown in Fig. 2. After single administration, mulberry fruits at all dosage range used in this study significantly decreased escape latency in alcoholic rat $(\mathrm{p}<0.001,0.001, .05$ respectively; compared with vehicle treated group). At 7 days after administration, the significant reduction of escape latencies were still observed $(\mathrm{p}<001.01$ and 0.05 respectively; compared with alcohol plus vehicle treated group). These changes were still observed when the treatment was prolonged further to 14 days ( $<<0.001$ all; compared with alcohol plus vehicle treated group). The alterations of retention time were also assessed and results were shown in Fig. 2. Unfortunately, no significant change was observed.

The neuroprotective effect of mulberry fruits in alcoholic rats: The neuroprotective effect against neurotoxicity induced by alcohol was also investigated and the results were shown in Fig. 3. The current results showed that Vitamin $\mathrm{C}$, the well known antioxidant, significantly increased the density of neurons in CA1, CA2 and dentate gyrus of the alcoholic rat $(\mathrm{p}<0.01$, 0.05 and 0.001 respectively; compared with vehicle treated group). Mulberry fruits at dose of $2 \mathrm{mg} \mathrm{kg}^{-1}$ BW significantly increased the neurons density in CA1, CA2, CA3 and dentate gyrus $(\mathrm{p}<0.01,0.05,0.05$ and 0.001 respectively; compared with vehicle treated group) while the mulberry fruits at higher doses (10 and $50 \mathrm{mg} \mathrm{kg}$ BW) could increase the neuron density only in CA1 and dentate gyrus $(\mathrm{p}<0.001$ all; compared with vehicle treated group). 


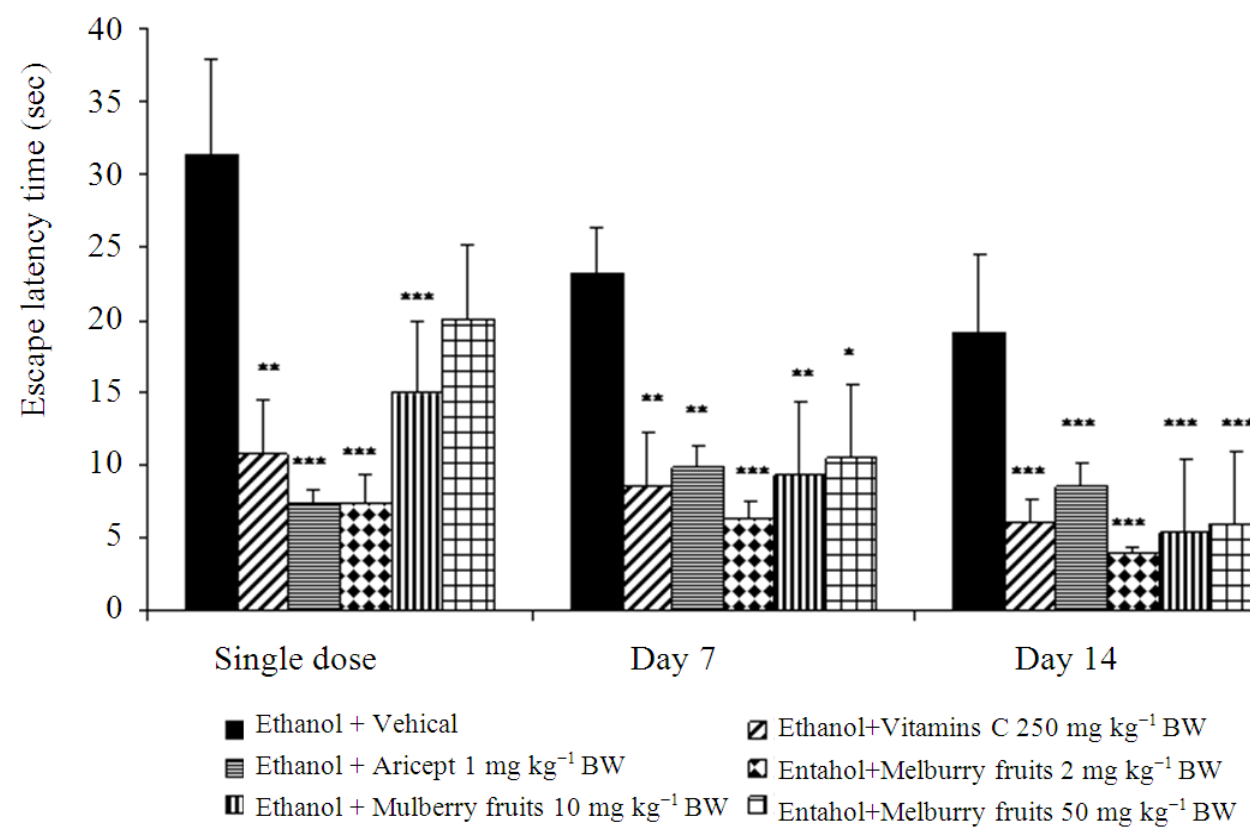

Fig. 1: Effect of Vitamin C, Aricept and various doses of mulberry fruits on escape latency time in Morris water maze test. Values given are the mean \pm S.E.M. $(n=6)^{*} \mathrm{p}<0.05$ as compared with vehicle plus ethanol.**p<0.01 as compared with vehicle plus ethanol. *** $\mathrm{p}<0.001$ as compared with vehicle plus ethanol

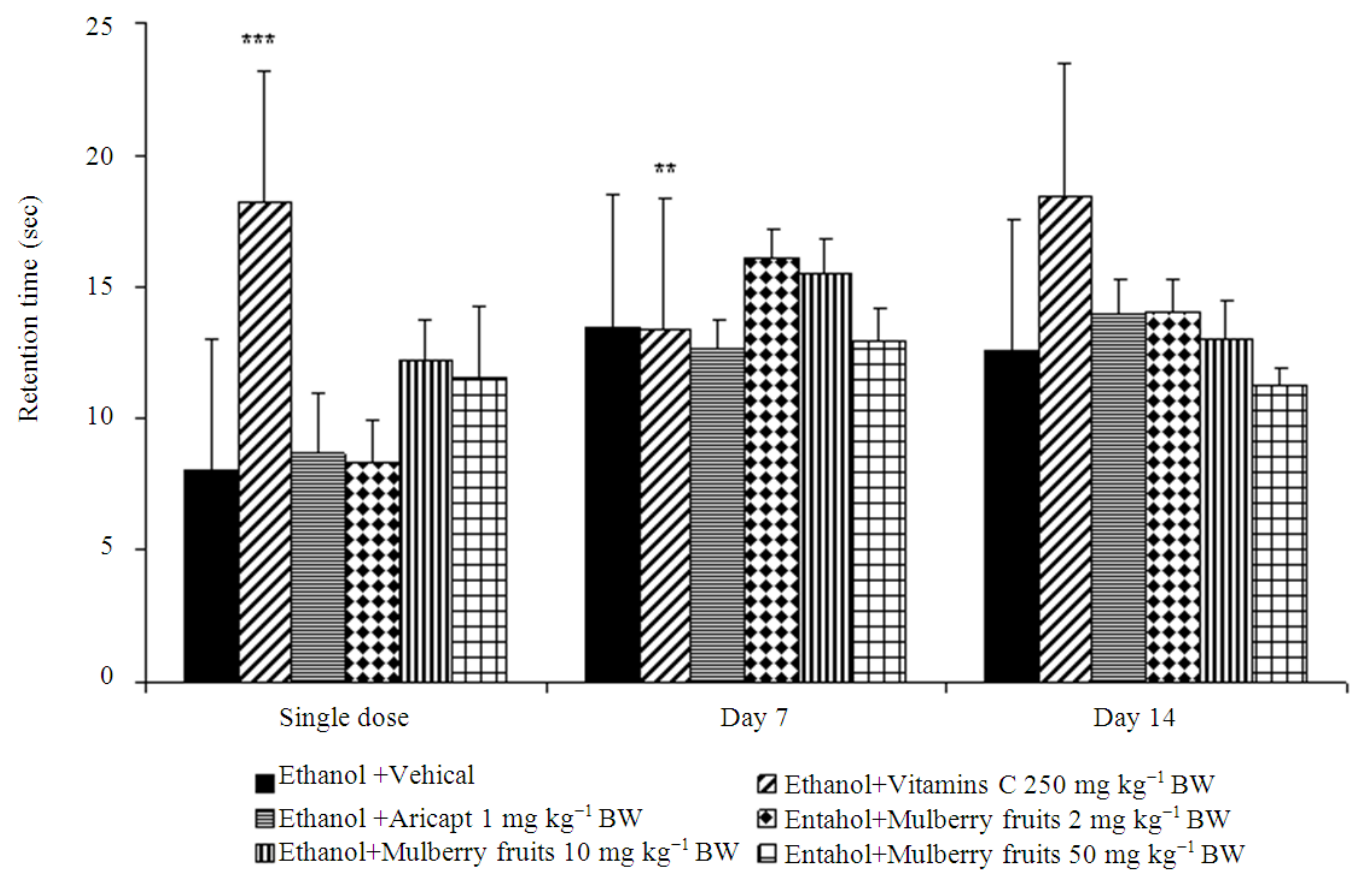

Fig. 2: Effect of Vitamin C, Aricept and various doses of mulberry fruits on retention time in Morris water maze test. Values given are the mean \pm S.E.M. $(n=6) * \mathrm{p}<0.05$ as compared with vehicle plus ethanol. $* * \mathrm{p}<0.01$ as compared with vehicle plus ethanol. $* * * p<0.001$ as compared with vehicle plus ethanol 
Am. J. Applied Sci., 9 (4): 484-491, 2012

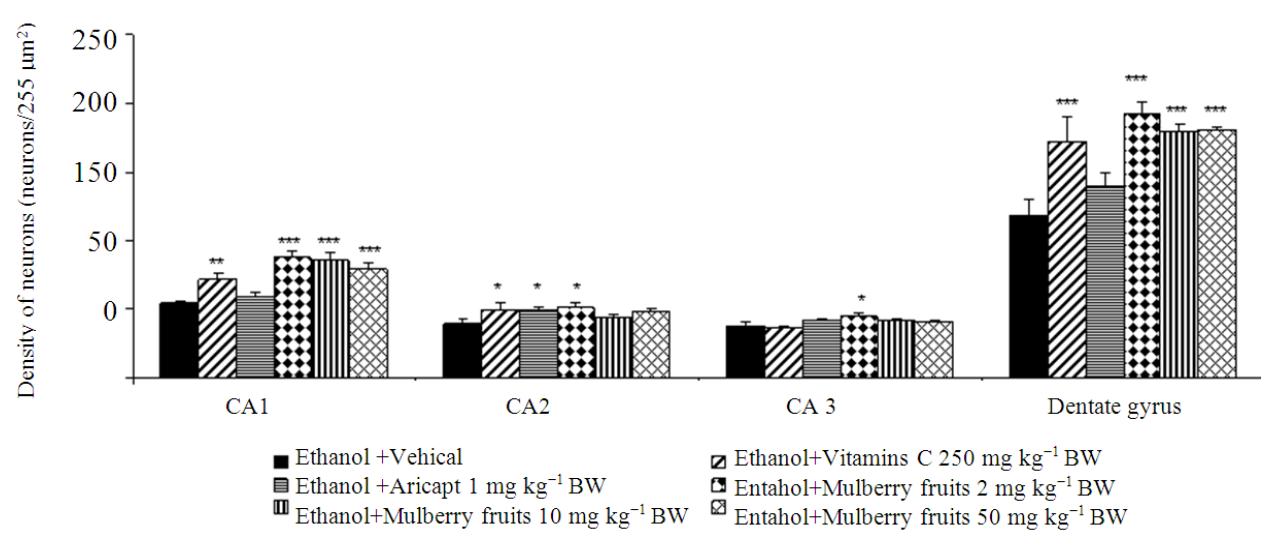

Fig. 3: Effect of Vitamin C, Aricept and various doses of mulberry fruits on neurons density in various subregions of hippocampus. Values given are the mean \pm S.E.M. $(n=6) * p<0.05$ as compared with vehicle plus ethanol. ** $\mathrm{p}<0.01$ as compared with vehicle plus ethanol. $* * * \mathrm{p}<0.001$ as compared with vehicle plus ethanol

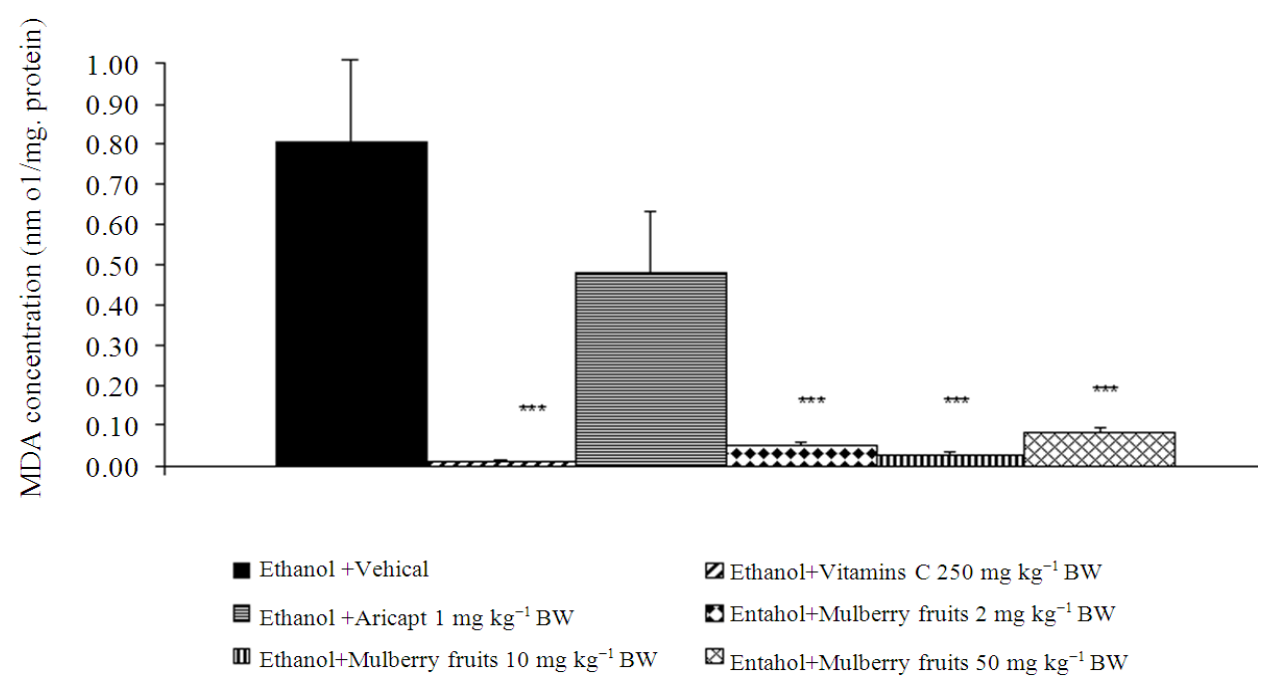

Fig. 4: Effect of Vitamin C, Aricept and various doses of mulberry fruits on the level of Malondialdehyde (MDA), a product of lipid peroxidation in hippocampus. Values given are the mean \pm S.E.M. $(n=6) * p<0.05$ as compared with vehicle plus ethanol. ** $\mathrm{p}<0.01$ as compared with vehicle plus ethanol. $* * * p<0.001$ as compared with vehicle plus ethanol

Effect of mulberry fruits on Malondialdehyde (MDA) level: Based on the previous information about the crucial role of oxidative stress on the neurodegeneration and the neuroprotective effect of mulberry fruits, the effect of mulberry fruits on oxidative stress damage marker, the Malondialdehyde (MDA) was determined. It was found that after 2 weeks of treatment, Vitamin C significantly decreased MDA level in hippocampus as shown in Fig. 4. Mulberry fruits at all doses used in this study also significantly decreased MDA level in the mentioned area $(\mathrm{p}<05$ all; compared with vehicle treated group).
Effect of mulberry fruits on Acetylcholinesterase (AChE) activity: Based on the previous information that cholinergic system played the crucial role on the memory impairment induced by chronic alcohol consumption, we also determined the activity of AChE activity in hippocampus. Figure 5 showed that Vitamin C significantly suppressed AChE activity ( $\mathrm{p}<0.05$; compared to vehicle treated group). In addition, mulberry fruits at dose of 2,10 and $50 \mathrm{mg}$ $\mathrm{kg}^{-1}$ BW also suppressed AChE activity $(\mathrm{p}<05.01$ and 0.05 respectively; compared to vehicle treated group). 
Am. J. Applied Sci., 9 (4): 484-491, 2012

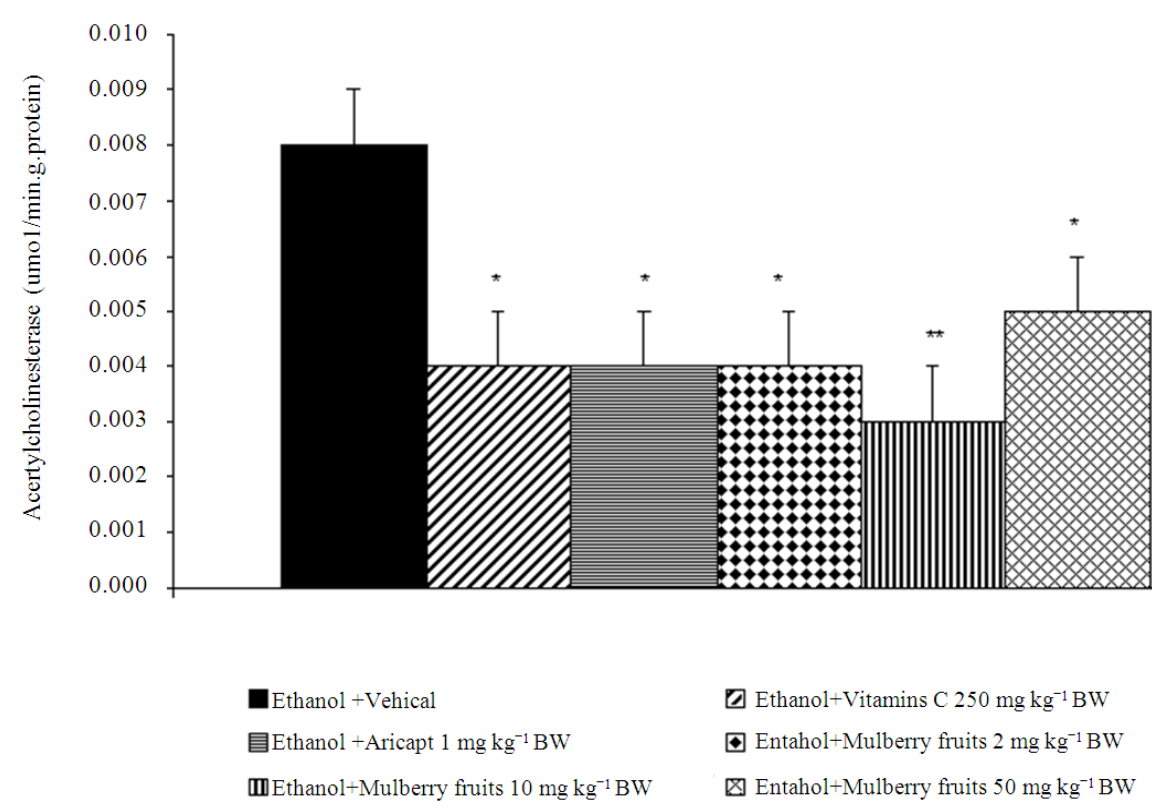

Fig. 5: Effect of Vitamin C, Aricept and various doses of mulberry fruits on the level of Acetylcholinesterase (AChE) in hippocampus. Values given are the mean \pm S.E.M. $(n=6)^{*} \mathrm{p}<0.05$ as compared with vehicle plus ethanol. ${ }^{* *} \mathrm{p}<0.01$ as compared with vehicle plus ethanol. ${ }^{* * *} \mathrm{p}<0.001$ as compared with vehicle plus ethanol

\section{DISCUSSION}

The current study demonstrated that mulberry fruits powder could mitigate memory impairment in animal model of alcoholism. In addition, mulberry fruits also increased neuron density in hippocampus in accompanied with the suppression of AChE activity and MDA level.

Brain has been reported to be susceptible to lipid peroxidation, since it consumes a large amount of oxygen and is rich in polyunsaturated fatty acids, which are especially prone to reactive oxygen injury. Oxidative stress appears to be one key factor inducing the pathogenesis of alcohol related brain damage (Gotz et al., 2001; Montoliu et al., 1994; Sun et al., 1997). Previous study had demonstrated that chronic ethanolinduced increases in CYP2E1 and other oxidases resulting in the increased lipid peroxidation and reactive oxygen radicals in the brain (Montoliu et al., 1994; Crew, 1999).

Recent studies have shown that oxidative related damage including brain damage and memory impairment can be prevented and attenuated by polyphenolic compounds which exhibited potent antioxidant activity (Rodrigo et al., 2011; Kim et al., 2010; Spencer, 2010; Sun et al., 2002). In addition, mulberry fruit extract have been previously reported to exhibit the neuroprotective effect via its antioxidant activity leading to the decreased oxidative stress and finally decreased neurodegeneration (Kim et al., 2010). These lines of evidence are in agreement with our finding that the mulberry fruits powder used in this study contained abundant of polyphenol and anthocyanin and it could decrease oxidative stress damage indicating by the reduction of MDA level in the hippocampus. Therefore, the neuroprotective effect of mulberry fruits observed in this study might occur partly via its antioxidant effect which in turn gave rise to the decreased oxidative damage. However, other effect related to the neuroprotection induced by mulberry fruits such as anti-apoptotic effect was also reported (Kim et al., 2010). Therefore, the neuroprotection of mulberry fruits related to antiapoptotic effect still could not be excluded. The possible active ingredient might be associated with the content of polyphenol compounds including anthocyanin in the mulberry fruits powder.

Previous studies had clearly demonstrated that the memory impairment was related to the hippocampal damage (Goodrich-Hunsaker and Hopkins, 2010; Graham et al., 2006). Therefore, it might be possible that the mulberry fruits decreased oxidative damage in hippocampus resulting in the increased neuron density in hippocampus leading to the improved spatial memory impairment induced by alcoholism. In addition, it was reported that Acetylcholine (ACh) 
modulated the encoding and retrieval of spatial memory in hippocampus (Rogers and Kesner, 2003). Accumulating lines of evidence had suggested that drugs or natural products exhibiting Acetylcholinesterase inhibitor (AChEI) could enhance the available ACh resulting in the improved memory impairment in various conditions (Chonpathompikunlert et al., 2010; Wattanathorn et al., 2011; Yuede et al., 2007). Our results also showed that mulberry fruits powder suppressed AChE activity in hippocampus. Therefore, these findings suggested that the cognitive enhancing effect of mulberry fruits might occur partly via the AChE suppression.

The present results failed to show the dose dependent effect of both neuroprotection and cognitive enhancing effects induced by mulberry fruits because mulberry fruits contained numerous ingredients. Therefore, the increasing doses of mulberry fruits might also increase the concentration of other ingredients which in turn can mask the effect of active ingredient. In addition, it was also possible that the relationships between mulberry fruits and the interested parameters which included both memory and neuron density in hippocampus were not the simple relationship.

\section{CONCLUSION}

Mulberry fruits can provide the beneficial effect to protect against brain damage induced by alcoholism and can be served as the valuable functional food. However, further researches about possible active ingredients, precise underlying mechanism and chronic toxicity are still essential before move forward to clinical trial study to confirm the beneficial effect of mulberry fruit as functional food to protect against brain damage and memory impairment in alcoholism.

\section{ACKNOWLEDGEMENT}

This study was supported by the Integrative Complimentary Alternative Medicine, Khon Kaen University. The authors also would like to express sincere thank for all kindness help about mulberry fruits collection and policy facilitation derived from the administrative team of The Queen Sirikit Department of Sericulture, Ministry of Agriculture and Cooperatives, Thailand.

\section{REFERENCES}

Agartz, I., R. Momenan, R.R. Rawlings, M.J. Kerich and D.W. Hommer, 1999. Hippocampal volume in patients with alcohol dependence. Arch. Gen. Psychiatry, 56: 356-363. PMID: 10197833
Arendt, T., Y. Allen, R.M. Marchbanks, M.M. Schugens and J. Sinden et al., 1989. Cholinergic system and memory in the rat: Effects of chronic ethanol, embryonic basal forebrain brain transplants and excitotoxic lesions of cholinergic basal forebrain projection system. Neuroscience, 33: 435-462. PMID: 2636702

Chonpathompikunlert, P., J. Wattanathorn and S. Muchimapura, 2010. Piperine, the main alkaloid of Thai black pepper, protects against neurodegeneration and cognitive impairment in animal model of cognitive deficit like condition of Alzheimer's disease. Food Chem. Toxicol., 48: 798-802. DOI: 10.1016/j.fct.2009.12.009

Crew, F.T., 1999. Alcohol and neurodegeneration. CNS Drug Rev., 5: 379-394. DOI: 10.1111/j.15273458.1999.tb00112.x

Das, S.K. and D.M. Vasudevan, 2007. Alcohol-induced oxidative stress. Life Sci., 81: 177-187. DOI: 10.1016/J.LFS.2007.05.005

Esterbauer, H., R.J. Schaur and H. Zollner, 1991. Chemistry and biochemistry of 4-hydroxynonenal, malonaldehyde and related aldehydes. Free Radic. Biol. Med., 11: 81-128. DOI: 10.1016/08915849(91)90192-6

Gong, S.X. and J.P. Zhu, 2008. Mulberry relieving nutritional anemia. Zhe Jiang Zhong Yi Yao Da Xue Xue Bao., 32: 350-352.

Goodrich-Hunsaker, N.J. and R.O. Hopkins, 2010. Spatial memory deficits in a virtual radial arm maze in amnesic participants with hippocampal damage. Behav. Neurosci., 124: 405-413. DOI: 10.1037/a0019193

Gotz, M.E., B. Janetzky, S. Pohli, A. Gottschalk and W. Gsell et al., 2001. Chronic alcohol consumption and cerebral indices of oxidative stress: Is there a link? Alcohol.: Clin. Exp. Res., 25: 717-725. DOI: $10.1111 / j .1530-0277.2001 . t b 02272 . x$

Graham, K.S., V.L. Scahill, M. Hornberger, M.D. Barense and A.C.H. Lee et al., 2006. Abnormal categorization and perceptual learning in patients with hippocampal damage. J. Neurosci., 26: 75477554. DOI: 10.1523/JNEUROSCI.1535-06.2006

Kang, T.H., J.Y. Hur, H.B. Kim, J.H. Ryu and S.Y. Kim, 2006. Neuroprotective effects of the cyanidin-3-O- $\beta$-d-glucopyranoside isolated from mulberry fruit against cerebral ischemia. Neurosci. Lett., 391: 122-126. DOI: 10.1016/J.NEULET.2005.08.053

Kim, H.G, M.S. Ju, J.S. Shim, M.C. Kim and S.H. Lee et al., 2010. Mulberry fruit protects dopaminergic neurons in toxin-induced Parkinson's disease models. Br. J. Nutr., 104: 8-16. DOI: $10.1017 /$ S0007114510000218 
Li, S. and X. Luo, 2003. Compendium of Materia Medica. 1st Edn., Foreign Languages Press, Beijing, ISBN: 7119032607, pp: 4397.

Ma, Y.P., 2002. Clinical observation of mulberry as a medicine of pharyngitis treatment. Xinjiang $\mathrm{J}$. Tradit. Chinese Med., 20: 83-84.

Matthews, D.B. and A.L. Morrow, 2000. Effects of acute and chronic ethanol exposure on spatial cognitive processing and hippocampal function in the rat. Hippocampus, 10: 122-130. DOI: $10.1002 /($ SICI)10981063(2000)10:1<122::AID-HIPO13>3.0.CO;2-V

Montoliu, C., S. Valles, J. Renau-Piqueras and C. Guerri, 1994. Ethanol-induced oxygen radical formation and lipid peroxidation in rat brain: Effect of chronic alcohol consumption. J. Neurochem., 63: $1855-1862$. DOI: $10.1046 /$ j.14714159.1994.63051855.x

Nordberg, A., C. Larsson, E. Perdahl and B. Winblad, 1982. Cholinergic activity in hippocampus in chronic alcoholism. Drug Alcohol Depend., 10: 333-344. DOI: 10.1016/0376-8716(82)90035-7

Rodrigo, R., A. Miranda and L. Vergara, 2011. Modulation of endogenous antioxidant system by wine polyphenols in human disease. Clin. Chim. Acta, 412: 410-424. DOI: 10.1016/J.CCA.2010.11.034

Rogers, J.L. and R.P. Kesner, 2003. Cholinergic modulation of the hippocampus during encoding and retrieval. Neurobiol. Learn. Memory, 80: 332342. DOI: $10.1016 / \mathrm{S} 1074-7427(03) 00063-7$
Shih, P.H., Y.C. Chan, J.W. Liao, M.F. Wang and G.C. Yen, 2010. Antioxidant and cognitive promotion effects of anthocyanin-rich mulberry (Morus atropurpurea L.) on senescence-accelerated mice and prevention of Alzheimer's disease. J. Nutr. Biochem., 21: 598-605. DOI: 10.1016/j.jnutbio.2009.03.008

Spencer, J.P.E., 2010. The impact of fruit flavonoids on memory and cognition. Br. J. Nutr., 104: S40-S47. DOI: $10.1017 /$ S0007114510003934

Sun, A.Y., A. Simonyi and G.Y. Sun, 2002. The "French paradox" and beyond: Neuroprotective effects of polyphenols. Free Radic Biol. Med., 32: 314-318. DOI: 10.1016/S0891-5849(01)00803-6

Sun, A.Y., Y.M. Chen, M. James-Kracke, P. Wixom and Y. Cheng, 1997. Ethanol-induced cell death by lipid peroxidation in PC12 cells. Neurochem. Res., 22: 1187-1192. PMID: 9342721

Wattanathorn, J., J. Jittiwat, T. Tongun, S. Muchimapura and K. Ingkaninan, 2011. Zingiber officinale mitigates brain damage and improves memory impairment in focal cerebral ischemic rat. Evid. Based Complement Alternat. Med., 2011: 429505-429505. DOI: 10.1155/2011/429505

Yuede, C.M., H. Dong and J.G. Csemansky, 2007. Anti-dementia drugs and hippocampal-dependent memory in rodents. Behav. Pharmacol., 18: 347363. DOI: 10.1097/FBP.0b013e3282da278d 